\title{
ON THE LASER-INDUCED MODIFICATION OF THE ENERGY BAND STRUCTURE
}

\author{
J.Z. KAMIŃSKI \\ Institute of Theoretical Physics, Warsaw University, Hoża 69, 00-681 Warszawa, Poland* \\ (Received November 25, 1992)

\begin{abstract}
We present a general method for the calculation of the energy band structure for a one-dimensional Kronig-Penney potential in the presence of a strong laser field. Numerical illustrations show that the energy band structure is strongly modified by the intense radiation field. In particular, it is also shown that the laser field creates new laser-induced forbidden gaps, widths of which increase with increasing intensity of radiation
\end{abstract}

PACS numbers: $71.35 .+\mathrm{z}$

\section{Introduction}

In recent years we have seen the development of technological methods to produce systems of reduced dimensionality. These systems can be used to study qualitatively new effects, the range of which is amazing indeed, and which have a profound impact on both fundamental theory and device physics. An example of such systems is the superlattice, i.e., a sequence of alternating ultra-thin epitaxial layers, in which a one-dimensional potential is superimposed on the background crystal potential [1]. While the crystal potential has the same period as the lattice centers, the period of the superlattice potential is the same as the alternating layers. Moreover, solid-state chemists can prepare isolated very thin metallic wires with diameters of only $6 \AA$, i.e., of atomic dimensions; these systems are going to be investigated with the help of far-infrared electromagnetic radiation [2].

A central theme of solid state physics is the study of electronic properties of matter. These properties depend on chemical bondings that come from the attraction of electrons to the positively charged nuclei in the relevant configuration, and the Coulomb repulsion because electrons are charged. When chemical bondings dominate the electron properties are well modeled by the energy band structure theory. In this work we are interested in this case.

The detailed nature of the energy bands may be worked out, in principle, by solving the Schrödinger equation provided that the crystal potential is known. The simplest model for band structure calculations is the Kronig-Penney

*Electronic address: jkam@fuw.edu.pl. 
model. In this model, the periodic potential is approximated by the periodic square wells. This allows one to solve easily the Schrödinger equation, and to show that the discrete atomic energy levels broaden into bands in solids [3]. In particular, the Kronig-Penney models have been applied to calculate the bands in a superlattice, and had considerable success in describing the optical properties of $\mathrm{GaAs} / \mathrm{Ga}_{1}-{ }_{-x} \mathrm{Al}_{x} \mathrm{As}$ superlattices [4]. These models have many advantages, for instance, they are relatively simple and easy to implement.

On the other hand, a new field of fundamental interest is opened by the observation of laser-induced variations of semiconductor properties [5]. Moreover, the appearance of lasers capable of generating high-power far-infrared coherent radiation has revealed new aspects of the multiphoton interaction of electromagnetic radiation with solids, where strong modifications of quantum processes by an intense far-infrared laser field can been expected [6]. These modifications are due to the fact that the effective coupling of radiation to matter can qualitatively be characterized by a dimensionless parameter $\eta=\left(I / I_{0}\right)\left(\omega / \omega_{0}\right)^{-3}$, where the intensity $I$ and the laser photon energy $\omega$ are in units of $\mathrm{W} \mathrm{cm}{ }^{-2}$ and $\mathrm{eV}$, respectively, $I_{0}=3.5 \times 10^{16} \mathrm{~W} \mathrm{~cm}^{-2}$ and $\omega_{0}=27.2 \mathrm{eV}$ [7]. This means that the softer laser photons, the smaller intensities of radiation are necessary in order to observe non-perturbative effects that appear for $\eta$ of the order of 1 .

The laser-induced reconstruction of band structure in semiconductors has been a subject of long-standing efforts, beginning, as far as we know, with the seminal work by Yelesin [8] (see, also [9]). The condensation of excitons in the presence of a strong coherent electromagnetic field has been studied in [10], where it has been shown that the density of excitons non-monotonically depends on both the intensity and the frequency of electromagnetic radiation, leading to hysteresis in both these parameters. This means that the interaction of a strong laser field with semiconductors leads to the optical bistability, which has been intensively studied during last years both experimentally [11] and theoretically [11,12]. Other aspects of the interaction of laser beams with semiconductor superlattices and quantum wells are considered for instance in [13] and references therein.

This paper is organized as follows. In Sec. 2 we set up a general mathematical scheme for the modeling of strong electromagnetic field effects on semiconductors, in particular for semiconductor superlattices. It should be noted, however, that most of the current experimental results, including those quoted before, do not refer to the superlattice case, but rather to bulk semiconductor of multiple quantum well systems. The long-range periodicity of the superlattice is therefore not essential. Nevertheless, we further apply this scheme to the calculation of band structure in Sec. 3. Other possible applications of the method presented in this paper are under considerations now. In our investigations the semiconductor superlattice is modeled by a one-dimensional Kronig-Penney potential. This means that only valence band is considered. On the other hand, it is known that the most interesting optical transitions connect valence and conduction bands. The approach presented in this paper can be generalized in order to encompass also these intraband transitions. However, for the sake of clarity such complications are not considered. The last section contains some closing remarks and prospects for further investigations. 


\section{The method}

The aim of this section is to develop the method which allows one to determine band structure for an arbitrary potential, which is constant in finite intervals. Such a potential can be described by two sets of real numbers: $\left\{x_{i}\right\}_{i=0,1} \ldots, K-1$ and $\left\{V_{i}\right\}_{i=0,1, \ldots, K} ; V_{i}$ is the value of the potential in the interval $\left[x_{i-1}, x_{i}\right]$. It is well known from textbooks how to treat the radiationless problem for such potentials. Namely, one divides the space into $K$ non-overlapping domains and for each of these domains one writes down the most general form of the wave function, the explicit form of which is determined from the matching conditions (i.e., fróm the continuity of the wave function and of its space derivative) and from Bloch's periodicity condition. It appears that a similar method can be applied to the analysis of band structure in the presence of electromagnetic plane waves. However, in order to do this we must know the most general solution of the time-dependent Schrödinger equation (the units in which $\hbar=c=1$ are used here)

$$
\mathrm{i} \partial_{t} \psi=\left[-\frac{1}{2 m} \frac{\partial^{2}}{\partial x^{2}}+\frac{\mathrm{i} e}{m} A(t) \frac{\partial}{\partial x}+V(x)\right] \psi
$$

in domains of constant $V(x)$. In this equation the vector potential $A(t)$ describes an electromagnetic plane wave in the dipole approximation

$$
A(t)=\mathcal{A} \cos (\omega t)
$$

and $V(x)$ is a static potential which is aimed to model the crystal lattice. Note that we have neglected the $A^{2}(t)$-term which can be eliminated by the space-independent unitary transformation, hence, it does not change the band structure. Moreover, we assume for simplicity that effective masses are the same for all intervals $\left[x_{i-1}, x_{i}\right]$.

We shall now construct a general solution of (1). By virtue of the fact that the Hamiltonian in (1) is periodic in time, the most general solution of the Schrödinger equation (1) can be build up of the Floquet-type wave functions [14],

$$
\psi(x, t)=\mathrm{e}^{-\mathrm{i} E t} \Phi(x, t),
$$

where $E$ is the quasi-energy and the function $\Phi$ is periodic in time, i.e.,

$$
\Phi(x, t)=\Phi(x, t+2 \pi / \omega) .
$$

Let us note, in passing, that due to the periodicity of $\Phi$ the quasi-energy $E$ is not defined uniquely. Indeed, the transformation (for integer $n$ )

$$
\begin{aligned}
& E \longrightarrow E+n \omega, \\
& \Phi(x, t) \longrightarrow \mathrm{e}^{\mathrm{i} n \omega t} \Phi(x, t)
\end{aligned}
$$

lea ves the Floquet state (3) invariant. Hence, the quasi-energy $E$ is defined modulo $\omega$, or in other words, the quasi-energy spectrum can be divided into an enumerable number of Brillouin zones of widths being equal to $\omega$.

The time-periodic function $\Phi$ fulfills the partial differential equation which we write as,

$$
\left[E+\mathrm{i} \partial_{t}+\frac{1}{2 m} \frac{\partial^{2}}{\partial x^{2}}-\frac{\mathrm{i} e}{m} A(t) \frac{\partial}{\partial x}-V(x)\right] \Phi=0 .
$$


Applying now the unitary transformation

$$
\Phi(x, t)=\exp \left[-\alpha_{0} \sin (\omega t) \frac{\partial}{\partial x}\right] \tilde{\Phi}(x, t)=\tilde{\Phi}\left[x-\alpha_{0} \sin (\omega t), t\right],
$$

we find that $\tilde{\Phi}$ fulfills the following equation:

$$
\left\{E+\mathrm{i} \partial_{t}+\frac{1}{2 m} \frac{\partial^{2}}{\partial x^{2}}-V\left[x+\alpha_{0} \sin (\omega t)\right]\right\} \tilde{\Phi}=0,
$$

where $\alpha_{0}=-e \mathcal{A} / m \omega$. This equation characterizes in fact the dynamics in a moving frame of reference which follows the quiver motion of the classical electron, and in which the crystal lattice oscillates.

A general solution of this equation for a constant in the whole space potential, $V(x)=V_{i}$, can easily be constructed. Accounting for the periodicity condition (4), such a solution adopts the form ( $\mathcal{Z}$ means the set of integers),

$$
\tilde{\Phi}(x, t)=\sum_{n \in \mathcal{Z}} \mathrm{e}^{-\mathrm{i} n \omega t}\left(\mathrm{e}^{\mathrm{i} p_{i n} x} C_{i n}^{+}+e^{-i p_{i n} x} C_{i n}^{-}\right),
$$

where

$$
p_{\text {in }}=\left[2 m\left(E-V_{i}+n \omega\right)\right]^{1 / 2}
$$

with arbitrary complex constants $C_{i n}^{+}$and $C_{i n}^{-}$. Going back to the wave function $\psi$, we arrive at the following form of a general solution of (1), which satisfies the periodicity condition (4):

$$
\begin{aligned}
\psi(x, t)= & \mathrm{e}^{-\mathrm{i} E t} \sum_{n, N \in \mathcal{Z}} \mathrm{e}^{-\mathrm{i} N \omega t}\left[J_{-(N-n)}\left(\alpha_{0} p_{i n}\right) \mathrm{e}^{\mathrm{i} p_{i n} x} C_{i n}^{+}\right. \\
& \left.+J_{N-n}\left(\alpha_{0} p_{i n}\right) \mathrm{e}^{-\mathrm{i} p_{i n} x} C_{i n}^{-}\right], \quad x_{i-1}<x<x_{i},
\end{aligned}
$$

where we have used the well-known form of the generating function of the Bessel functions $J_{k}(z)$. This is the most general solution of the Schrödinger equation (1), which satisfies the Floquet conditions (3) and (4). It is easily seen that for the radiationless problem, when $\alpha_{0}=0$ and $N=0$, this solution reduces to the superposition of two counterpropagating plane waves of energy $E$, as it should be. However, for a non-vanishing laser field the structure of this solution is much more complicated, because instead of two arbitrary constants it contains now an infinite number of them, what makes computations much more difficult. Moreover, this solution contains the so-called closed channels, for which the momenta $p_{\text {in }}$ defined by (11) become complex numbers. It is well known from the scattering theory [15] that such closed channels - although not "visible" - play a very important role in quantum mechanics; for instance one cannot satisfy the probability conservation law without taking them into account. Of course, in numerical calculations the infinite sums over $n$ and $N$ have to be truncated. This problem is going to be discussed shortly. form

The matching conditions for $x=x_{i-1}$ can be written down in the matrix

$$
N\left(i-1, x_{i-1}\right) C_{i-1}=N\left(i, x_{i-1}\right) C_{i},
$$

in which

$$
N(i, x)=\left(\begin{array}{cc}
N^{+}(i, x) & N^{-}(i, x) \\
N^{+}(i, x) & N^{-}(i, x)
\end{array}\right), \quad C_{i}=\left(\begin{array}{c}
C_{i}^{+} \\
C_{i}^{-}
\end{array}\right)
$$


and

$$
\begin{aligned}
& C_{i}^{ \pm}(n)=C_{i n}^{ \pm}, \\
& {\left[N^{ \pm}(i, x)\right]_{N, n}=J_{ \pm(n-N)}\left(\alpha_{0} p_{i n}\right) \mathrm{e}^{ \pm \mathrm{i} p_{i n} x}} \\
& {\left[N^{ \pm}(i, x)\right]_{N, n}= \pm \mathrm{i} p_{i n} J_{ \pm(n-N)}\left(\alpha_{0} p_{i n}\right) \mathrm{e}^{ \pm p_{i n} x} .}
\end{aligned}
$$

Hence, we arrive at the following chain of equations for the columns $C_{i}$ :

where

$$
C_{i}=N_{i} C_{i-1}
$$

$$
N_{i}=\left[N\left(i, x_{i-1}\right)\right]^{-1} N\left(i-1, x_{i-1}\right) .
$$

This chain of equations allows one to connect the solution for $x_{i-1}<x<x_{i}$ to the solution for $x_{j-1}<x<x_{j}$,

$$
C_{j}=N_{j} N_{j-1} \ldots N_{i+1} C_{i}=\mathcal{T}_{j i} C_{i},
$$

where the matrix $\mathcal{T}_{j i}$ is called the transfer matrix.

Let us assume now that $V(x)$ is a periodic function of $x$ with the period equal to $l$. This means that there exists such an index $P$ that $V_{i}=V_{i+P}=V_{i+2 P}=\ldots$, and $x_{i+P}=x_{i}+l$. Hence, we know from the Bloch theorem that for a given time $t$ a general solution of (1) can be built up of the Bloch-type wave functions of the form $[16]$

$$
\psi(x, t)=\mathrm{e}^{\mathrm{i} q x} u_{q}(x, t)
$$

where $q$ is the quasi-momentum and $u_{q}(x+l, t)=u_{q}(x, t)$. Similarly to the case of quasi-energies, the quasi-momentum is defined modulo $2 \pi / l$; in our further considerations we assume that $q \in[-\pi / l, \pi / l]$. The Bloch theorem and equations (17) and (12) lead us to the following "self-consistent" eigenvalue equation:

$$
\mathcal{F}_{i, P} C_{i}=\mathrm{e}^{\mathrm{i} q l} C_{i}
$$

in which the "Bloch-Floquet" matrix is defined as

$$
\mathcal{F}_{i, P}=\mathcal{P}_{i} \mathcal{T}_{i+P, i}
$$

where

$$
\mathcal{P}_{i}=\left(\begin{array}{cc}
\mathcal{P}_{i}^{+} & 0 \\
0 & \mathcal{P}_{i}^{-}
\end{array}\right)
$$

and $\left(\mathcal{P}_{i}^{ \pm}\right)_{N n}=\delta_{N n} \exp \left( \pm i p_{i n} l\right)$. This is the eigenvalue equation which determines the dispersion relation $E=E(q)$ and the coefficients $C_{i}$ necessary for the construction of the explicit form of the wave functions (12).

The eigenvalue equation (19) consists of an infinite system of linear algebraic equations. In practical calculations such a system has to be truncated. This means that the indices $N$ and $n$ in (12) run over a finite subset of integers $\mathcal{Z}$. Of course, such a truncation also reduces the number of eigenvalues and modifies their numerical values. Therefore, in our further numerical analysis we carry out the calculation for two subsets of integers, $\mathcal{Z}_{0}$ and $\mathcal{Z}_{1}$, such that $\mathcal{Z}_{0} \subset \mathcal{Z}_{1}$, and choose for a given quasi-energy $E$ such eigenvalues of quasi-momentum $q$ that within a prescribed error (in this paper $10^{-3}$ ) do not change their numerical values. We take for $\mathcal{Z}_{0}$ and $\mathcal{Z}_{1}$ a subset of integers changing from $-n_{\min }$ to $n_{\max }$. The positive numbers $n_{\min }$ and $n_{\max }$ are chosen in such a manner that physical quantities under considerations become stable with respect to small changes of them. 


\section{Numerical illustrations}

The results of our numerical analysis are presented in the attached figures. In this analysis the Kronig-Penney potential is defined as

$$
V(x)= \begin{cases}V_{0}, & -a / 2<x<a / 2 \\ 0, & a / 2<x<b+a / 2\end{cases}
$$

with $a+b=l$. The numerical values of parameters are: $a=1, b=10$ and $V_{0}=1$. The absolute values of these parameters are not important because the results are invariant with respect to the following scaling relations:

$$
\left(a, b, \omega, \mathcal{A}, V_{0}, E, q\right) \longrightarrow\left(\lambda a, \lambda b, \lambda^{-2} \omega, \lambda^{-1} \mathcal{A}, \lambda^{-2} V_{0}, \lambda^{-2} E, \lambda^{-1} q\right) .
$$

This means that this potential describes for instance the superlattice with $a=$ $8.3 \AA, b=83 \AA$ and $V_{0}=0.1 \mathrm{eV}$. Moreover, the laser field is characterized by its frequency $\omega=0.3$ and by the dimensionless parameter $\lambda_{s c}=\mathcal{A} \omega^{-1 / 2}$, which determines the intensity of the laser field. For instance, for $\lambda_{\mathrm{sc}}=1$ and $\omega=0.03 \mathrm{eV}$ the intensity $I$ is equal to $4.7 \times 10^{7} \mathrm{Wcm}^{-2}$.

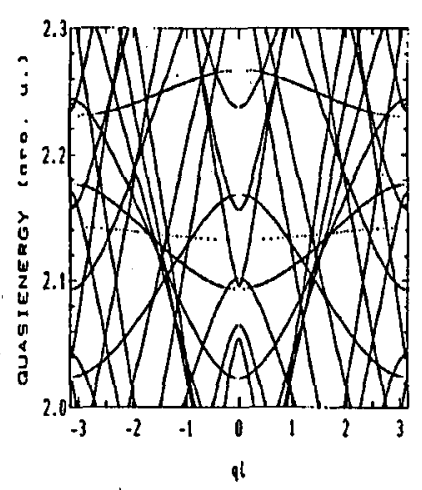

Fig. 1. The radiationless energy band structure for the Kronig-Penney potential (22) and for energy changing from 2 to 2.3. In this calculation the integers $N$ and $n$ change from -10 to 10 . Quasi-momenta $q$ are calculated with the help of the eigenvalue equation (19) for quasi-energies $E=2+0.0003 \times k, 0 \leq k<1000$, and for $\omega=0.3, \alpha_{0}=0$. This method is equivalent to the standard calculation of the band structure for energies changing from 0 to 5.3 (i.e., from $\max (0,2-10 \omega)$ to $2.3+10 \omega$ ), and subsequently, to the projection of the results on the interval [2, 2.3] modulo 0.3 (see the text for more explanations). Quasi-energies (in arbitrary units) are expressed as functions of the dimensionless parameter $g l$.

It is seen from Fig. 1 that for the zero intensity of the laser field we reproduce the radiationless energy band structure projected on the one Brillouin zone; we have already mentioned in the previous section that the quasi-energy is defined modulo $\omega$. In particular, we observe many crossings of $(E, q)$-curves. With increasing intensity (Figs. 2 and 3 ) some of these crossings transform themselves into a voided crossings, the widths of which increase with the intensity. Hence, the dispersion relation $E=E(q)$ is not further described by a monotonous function 

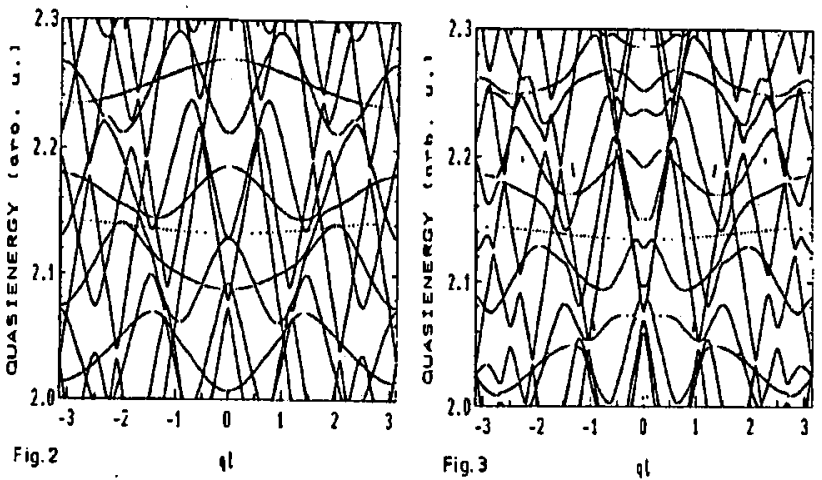

Fig. 2. The energy band structure for the Kronig-Penney potential (22) and for energy changing from 2 to 2.3 , in the presence of the laser field characterized by its frequency $\omega=0.3$ and by the dimensionless parameter $\lambda_{\mathrm{sc}}=\mathcal{A} \omega^{-1 / 2}=0.2$. The integers $N$ and $n$ change both from -12 to 12 in the first run, and from -17 to 17 in the second run. For a given quasi-energy $E=2+0.0003 \times k, 0 \leq k<1000$, we choose such quasi-momenta $q$, which are stable with the error smaller than $10^{-3}$.

Fig. 3. The same as in Fig. 2, but for $\lambda_{\mathrm{sc}}=0.5$. The integers $N$ and $n$ change from -15 to 15 , and from -20 to 20 .

of $q$, but exhibits many extremes. This is in contrast to the radiationless problem where it is well known that in the one-dimensional case the minima or maxima of $E(q)$ may occur only at $q=0$ or $q= \pm \pi / l$ (see, e.g., the footnote on page 79 in [17]). The avoided crossings in the band structure may cause strong modifications of optical properties of the electron gas in the presence of strong laser fields, because they lead to the resonant transitions between bands. Let us emphasize, however, that this problem is beyond the scope of this paper, the main purpose of which was to present a general method for the calculation of the band structure and the corresponding Bloch wave functions by solving the eigenvalue equation (19). In order to calculate other properties of semiconductor superlattices, modeled by the Kronig-Penney potential or its generalizations, one has to carry out far-reaching and time-consuming numerical investigations, which are under considerations now. Moreover, it is clearly seen that at the avoided crossings we observe the appearance of new laser-induced forbidden gaps, the widths of which depend on the intensity of the laser field. A similar effect also appears for the one-dimensional chain of atoms, in which the position of each atom is slightly distorted in such a way that the translational symmetry of the chain is reduced. Such a distortion is responsible for the appearance of forbidden gaps, which are often called the Peierls gaps [17] (see [18] for more details). We see that not only the distorted periodicity in space but also the extra periodicity in time, which is induced by coherent radiation, can create new forbidden gaps. It seems, therefore, natural to call them the optical Peierls gaps.

It may not be clear what the dotted lines on these figures mean. In order to explain this let us remind that in our method quasi-momenta $q$ are calculated 
from the eigenvalue equation (19) for a discrete set of quasi-energies $E$. This means that one can plot the continuous curves $E(q)$ provided that such a discretization is sufficiently dense and that the slope of $E(q)$ is not close to zero (otherwise a small change of $E$ creates a significant change of $q$ ). This is why the continuous curves at their extremes become dotted ones, as it is clearly seen for the lowest band near the well bottom, for which the dependence of $E$ on $q$ is rather marginal.

As it is especially seen from Fig. 3, there appear "unfinished" lines for sufficiently strong laser fields. This is a shortcoming of our stabilization method which consists in looking for such eigenvalues of (19) that are stable within a prescribed error with respect to the change of lower and upper limits of $n$ and $N$, as is described at the end of the previous section. These "unfinished" lines can easily be eliminated from figures. We have not done this in order to show that in these figures there exist "hidden" bands that are not explicitly present, because the truncation of the eigenvalue equation (19) has been too "drastic" for them, i.e., one should significantly increase the lower and upper limits of $N$ and $n$ in (12) in order to obtain full curves for these bands; for this, however, one needs much more computer time. On the other hand the information contained in these figures is not complete without knowing for instance the transition matrix elements, because it may appear that only few curves in these figures are relevant, and presumably all the "unfinished" lines are irrelevant. This problem is going to be addressed elsewhere.

Let us also make the following remarks closing this section. Figure 1 represents the energy band structure of the Kronig-Penney model in the absence of electromagnetic wave. This interpretation may appear to be unclear, because one can ask oneself: What is the relationship between the quasi-momentum $q$ and the actual energy $E$ of a particle? If truly the bands are defined modulo $\omega$, how is this picture rationalized with experimental evidence of clear interband optical transitions? As it has been explained previously the band structure is calculated by solving the eigenvalue equation (19), i.e., for a given energy $E$ we determine the "Bloch-Floquet" matrix $\mathcal{F}_{i, P}$, find the eigenvalues of this matrix and pick up the real values of quasi-momenta $q$. In this calculation we have two free integer parameters $n_{\min }$ and $n_{\max }\left(n_{\min }, n_{\max } \geq 0\right)$. This means that infinite columns $C_{i}^{ \pm}(k), k=-\infty, \ldots,+\infty$, in Eqs. (14) and (19) are truncated to the finite ones with $k=-n_{\min }, \ldots, n_{\max }$. Thus, by looking at (12), we see that the quasi-energy (which is defined modulo $\omega$ ) changes from $E-n_{\min } \omega$ to $E+n_{\max } \omega$. Since the energy of electrons moving in the periodic potential (22) cannot be smaller than zero, therefore, quasi-momenta $q$, determined by the truncated eigenvalue equation (19), correspond to electron energies changing from $\max \left(0, E-n_{\min } \omega\right)$ to $E+n_{\max } \omega$. This explains why Fig. 1 describes the band structure for energies from 0 to 5.3 projected on the interval $[2,2.3]$. How can we get from Fig. 1 the usual radiationless band structure? In order to do this we have also to know the truncated eigenvectors $C_{i}$. In the absence of electromagnetic field these eigenvectors have a very simple form; namely, except for one element, all elements of $C_{i}$ are equal to zero. This means that if $C_{i}^{+}\left(n_{0}\right) \neq 0$ (or $C_{i}^{-}\left(n_{0}\right) \neq 0$ ) then the quasi-momentum $|q|$ (or $-|q|$ ) corresponds to the energy $E+n_{0} \omega$, as it follows from the expression for the wave function (12). Hence, calculating the eigenvectors $C_{i}$ one can "un- 
fold" Fig. 1 and obtain the usual band structure. Such a procedure is still possible for weak electromagnetic fields. In this case there are many non-zero elements of eigenvectors $C_{i}$. However, there is only one non-vanishing element of $C_{i}$, the absolute value of which dominates, what again allows one to apply the method described before. Thus, as far as the radiation field is sufficiently weak, there is no contradiction between the physical pictures provided by both the standard perturbation theory with respect to the external radiation field and our method. Discrepancies between these two approaches start for strong electromagnetic field, i.e., when eigenvectors $C_{i}$ have many non-vanishing elements, and absolute values of at least two of them are comparable (this could be in fact the "operational" definition of a strong non-perturbative radiation field). For such fields the standard perturbation theory breaks down and the physical picture based on the sequential absorption or emission of single photons cannot longer be applied.

\section{Conclusions and prospects}

The use of a simple Kronig-Penney model, while not very quantitative, may be useful in giving qualitative physical insight into the effects of electromagnetic fields on the properties of semiconductors. The limitation to the qualitative aspects of the interaction of matter with strong external fields cannot be considered as a shortcoming of the method presented in this paper. This is due to the fact that in most cases the quantitative understanding of the interaction of strong external fields (that nowadays can be generated in laboratories) with matter is not possible. For example, a numerical computation of the spectrum and of different types of cross sections for such a simple system as the hydrogen atom in the presence of strong laser fields has not yet been attained. The aim of most of such studies is to understand rather qualitatively (and, if possible, also quantitatively) the enormous variety of phenomena that results from the interaction of electrons with both internal and external fields.

It is hoped that the method presented in this paper, together with its generalizations that also include the conduction bands, can be applied to investigations of such phenomena as the blue shift of the fundamental absorption edge and its dependence on intensity and detuning. Moreover, it would be interesting to know how intense radiation field modifies some exotic phenomena that appear in elementary quantum models with position-dependent mass [19].

\section{Acknowledgment}

The author wishes to thank Professor K. Dietz for his warm hospitality at the University in Bonn where this work has been initiated. This work has been supported by the Deutsche Sonderforschungsgemeinschaft (SFB - 334) and by the Polish Committee for Scientific Research (grant KBN 2042691 01).

\section{References}

[1] L.D. Smith, C. Mailhiot, Rev. Mod. Phys. 62, 173 (1990); L. Esaki, Int. J. Mod. Phys. B 3, 487 (1989); M.A. Herman, Semiconductor Superlatices, Academie-Verlag, Berlin 1986.

[2] F.J. DiSalvo, Science 247, 649 (1990). 
[3] S. Brandt, H.D. Dahmen, The Picture Book of Quantum Mechanics, Wiley, New York 1985.

[4] R. Dingle, W. Wiegmann, C.H. Henry, Phys. Rev. Lett. 33, 827 (1974); R. Dingle, A.C. Gossard, W. Wiegmann, Phys. Rev. Lett. 34, 1327 (1975).

[5] M. Joffre, D. Hulin, A. Migus, M. Combescot, Phys. Rev. Lett. 62, 74 (1989); M. Combescot, Phys. Rev. B 41, 3517 (1990); M. Combescot, R. Combescot, Phys. Rev. Lett. 61, 117 (1988); R. Binder, S.W. Koch, M. Lindberg, N. Peyghambarian, W. Schäfer, Phys. Rev. Lett. 65, 899 (1990); M. Lindberg, R. Binder, S.W. Koch, Phys. Rev. A 45, 1865 (1992); N. Peyghambarian, S.W. Koch, M. Lindberg, B. Fluegel, M. Joffre, Phys. Rev. Lett. 62, 1185 (1989); V. Langer, H. Stolz, W. van der Osten, Phys. Rev. Lett. 64, 854 (1990).

[6] J.Z. Kamiński, J. Phys. C, Solid State Phys. 21, 3983 (1988).

[7] J.Z. Kaminiski, Phys. Scr. 42, 417 (1990).

[8] V.F. Yelesin, Zh. Eksp. Teor. Fiz. 59, 602 (1970).

[9] V.M. Galitsky, S.P. Goreslavsky, V.F. Yelesin, Zh. Eksp. Teor. Fiz. 57, 207 (1969).

[10] V.F. Yelesin, Yu.V. Kopayev, Zh. Eksp. Teor. Fiz. 62, 1447 (1972).

[11] H.M. Gibbs, Optical Bistability: Controlling Light with Light, Academic Press, Orlando 1985.

[12] J. Goll, H. Haken, Phys. Status Solidi B 101, 480 (1980); C.M. Misra, P. Tripathi, G.S. Tripathi, Phys. Lett. A 117, 210 (1986).

[13] S. Datta, J. McLennan, Rep. Prog. Phys. 531003 (1990); E. Gharhramani, D.J. Moss, J.E. Sipe, Phys. Rev. Lett. 64, 2815 (1990); M.J. Kelly, R.J. Nicholas, Rep. Prog. Phys. 48, 1699 (1985); W.H. Knox, D.S. Chemla, D.A.B. Miller, J.B. Stark, S. Schmitt-Rink, Phys. Rev. Lett. 62, 1189 (1989); Y.J. Mii, K.L. Wang, R.P.G. Karunasiri, P.F. Yuh, Appl. Phys. Lett. 56, 1046 (1990); I. Morrison, M. Jaros, J. Phys, Condens. Malter 2, 4879 (1990); D. Paquet, Phys. Scr. Vol. T 23, 59 (1988); M.I. Stockman, L.N. Pandey, T.F. George, Phys. Rev. Lett. 65, 3433 (1990); C. Weisbuch, in: Physics and Applications of Quantum Wells and Superlattices, Eds. E.E. Mendez, K. von Klitzing, Plenum, New York 1987, p. 261; F. Yonezawa, K. Ishida, F. Sato, Int. J. Mod. Phys. B 3, 135 (1989).

[14] N.L. Manakov, V.D. Ovsiannikov, L.P. Rapoport, Phys. Rep. 141, 319 (1986); K. Yajima, Commun. Math. Phys. 87, 331 (1982); Ya. B. Zel'dovich, Zh. Eksp. Teor. Fiz. 51, 1492 (1966); V.I. Ritus, Zh. Eksp. Teor. Fiz. 51, 1544 (1966); T.H. Shirley, Phys. Rev. B 138, 979 (1965).

[15] J.R. Taylor, Scattering Theory, Wiley, New York 1972.

[16] N.W. Ashcroft, N.D. Mermin, Solid State Physics, Holt, Rinehart and Winston, New York 1976; B.K. Ridley, Quantum Processes in Semiconductors, Clarendon, Oxford 1982.

[17] R.E. Peierls, Quantum Theory of Solids, Clarendon, Oxford 1964.

[18] A.J. Berlinsky, Rep. Prog. Phys. 42, 1241 (1979); H. Böttger, Principles of the Theory of Lattice Dynamics, Academie-Verlag, Berlin 1983.

[19] J.-M. Lèvy-Leblond, Eur. J. Phys. 13, 215 (1992). 